\title{
INSIST-ED: Italian Society of Andrology registry on penile prosthesis surgery. First data analysis
}

\author{
Edoardo Pescatori ${ }^{1}$, Giovanni Alei ${ }^{2}$, Gabriele Antonini ${ }^{2}$, Antonio Avolio ${ }^{3}$, Carlo Bettocchi ${ }^{4}$, Marco Bitelli ${ }^{5}$, \\ Francesco Boezio ${ }^{6}$, Tommaso Cai ${ }^{7}$, Enrico Caraceni ${ }^{8}$, Maurizio Carrino ${ }^{9}$, Fulvio Colombo ${ }^{10}$, Enrico Conti ${ }^{11}$, \\ Antonio Corvasce ${ }^{12}$, Federico Dehò ${ }^{13}$, Stefano Fiordelise ${ }^{14}$, Nicola Ghidini ${ }^{1}$, Emilio Italiano ${ }^{15}$, \\ Giuseppe La Pera ${ }^{16}$, Giovanni Liguori ${ }^{17}$, Carlo Maretti ${ }^{18}$, Nicola Mondaini ${ }^{19}$, Alessandro Natali ${ }^{20}$, \\ Carlo Negro ${ }^{21}$, Alessandro Palmieri ${ }^{22}$, Fabrizio Palumbo ${ }^{4}$, Matteo Paradiso ${ }^{21}$, Massimo Polito ${ }^{23}$, \\ Diego Pozza ${ }^{24}$, Mauro Silvani ${ }^{25}$, Aldo Tamai ${ }^{26}$, Massimiliano Timpano ${ }^{27}$, Lilia Utizi ${ }^{8}$, Francesco Varvello ${ }^{28}$, \\ Patrizio Vicini ${ }^{29}$, Antonio Vitarelli ${ }^{4}$, Giorgio Franco ${ }^{2}$ \\ ${ }^{1}$ Hesperia Hospital, Modena, Italy; ${ }^{2}$ Policlinico Umberto I, Roma, Italy; ${ }^{3}$ Ospedale Mazzoni, Ascoli Piceno, Italy; ${ }^{4}$ Policlinico Bari, \\ Bari, Italy; ${ }^{5}$ Ospedale S. Sebastiano Martire, Frascati, Italy; ${ }^{6}$ Ospedale Tatarella, Cerignola, Italy; ${ }^{7}$ Ospedale Santa Chiara, Trento, \\ Italy; ${ }^{8}$ Area Vasta 3, Civitanova Marche, Italy; ${ }^{9}$ Ospedale Cardarelli, Napoli, Italy; ${ }^{10}$ Policlinico S.Orsola-Malpighi, Bologna, Italy; \\ ${ }^{11}$ Ospedale San Bartolomeo, Sarzana, Italy; ${ }^{12}$ Ospedale Bonomo, Andria, Italy; ${ }^{13}$ Ospedale San Raffaele, Milano, Italy; ${ }^{14}$ AUSL \\ Piacenza, Italy; ${ }^{15}$ Ospedali Riuniti, Palermo, Italy; ${ }^{16}$ San Camillo Forlanini, Roma, Italy; ${ }^{17}$ Ospedali Riuniti, Trieste, Italy; \\ ${ }^{18}$ CIRM, Piacenza, Italy; ${ }^{19}$ Ospedale S.M. Annunziata, Firenze, Italy; ${ }^{20}$ AUOC, Firenze, Italy; ${ }^{21}$ Ospedale Cardinal Massaia, Asti, Italy; \\ ${ }^{22}$ Università Federico II, Napoli, Italy; ${ }^{23}$ Ospedali Riuniti, Ancona, Italy; ${ }^{24}$ Wojtyla Hospital, Roma, Italy; ${ }^{25}$ Ospedale Infermi, Biella, \\ Italy; ${ }^{26}$ Casa di Cura Eretenia, Vicenza, Italy; ${ }^{27}$ Ospedale Molinette, Torino, Italy; ${ }^{28}$ Ospedale San Lazzaro, Alba, Italy; \\ ${ }^{29}$ Rome American Hospital, Roma, Italy.
}

\section{Summary Objectives: The Italian Society of Andrology, i.e. "Società Italiana di Andrologia" (S.I.A.), launched on} December 2014 a prospective, multicenter, monitored and internal review board approved Registry for penile implants, the "INSIST-ED" (Italian Nationwide Systematic Inventarisation of Surgical Treatment for ED) Registry. Purpose of this first report is to present a baseline data analysis of the characteristics of penile implant surgery in Italy. Material and methods: The INSIST-ED Registry is open to all surgeons implanting penile prostheses (all brands, all models) in Italy, providing anonymous patient, device, surgical procedure, outcome, follow-up data, for both first and revision surgeries. A Registry project Board overviews all the steps of the project, and a Registry Monitor interacts with the Registry implanting surgeons.

Results: As by April 8, 2016, 31 implanting surgeons actively joined the Registry, entering 367 surgical procedures in its database, that comprise: 310 first implants, 43 prosthesis substitutions, 14 device explants without substitution. Implanted devices account for: 288 three-component devices (81,3\%), 20 two-component devices (5,4\%), 45 non-hydraulic devices (12,3\%). Leading primary ED etiologies in first implant surgeries resulted: former radical pelvic surgery in 111 cases $(35,8 \%)$, Peyronie's disease in 66 cases $(21,3 \%)$, diabetes in 39 cases $(12,6 \%)$. Two intraoperative complications have been recorded. Main reasons for 57 revision surgeries were: device failure (52,6\%), erosion $(19,3 \%)$, infection $(12,3 \%)$, patient dissatisfaction (10,5\%). Surgical settings for patients undergoing a first penile implant were: public hospitals in 251 cases (81\%), private environments in 59 cases (19\%).

Conclusions: The INSIST-ED Registry represents the first European experience of penile prosthesis Registry. This baseline data analysis shows that: three-pieces inflatable prosthe- sis is the most implanted device, leading etiology of erectile dysfunction (ED) in patient receiving a prosthesis is former radical pelvic surgery, primary reason for revision surgery is device failure, primary settings for first penile implant surgery are public hospitals. Evaluation of penile implant impact on recipients quality of life is presently ongoing.

KEY WORDS: Penis; Penile prosthesis; Surgery; Surgical procedures; Implant; Registry; Quality of life; Impotence; Erectile dysfunction.

Submitted 30 April 2016; Accepted 6 May 2016

\section{INTRODUCTION}

Penile prosthetic surgery is a strategic and highly qualifying surgical tool for both patients with severe erectile dysfunction and Urologists devoted to Sexual Medicine.

The penile prosthesis option is the ED treatment presently reported to score the highest in terms of satisfaction among both patients and partners, compared to all the other available ED treatments $(1,2)$.

The paradox of the penile prosthesis is that, despite being an excellent solution for the patient with severe ED, majority of candidates to this treatment do not have access to it. In Italy the overall prevalence of ED in the adult population is $12,8 \%$ (3), accounting for about 3 million men. While a conservative estimate would predict not less than 200.000 men with severe ED, market data report that only roughly 500 penile implant surgeries are yearly performed in Italy. Several are the barriers to the penile prosthesis solution, among them paucity of proper patient information, and costs of penile implants: often public hospitals 
have no or few units/year available, with long waiting lists, while in private settings device costs are prohibitive for several patients. Furthermore, public Health Authorities seldom have reliable figures on the dimensions of penile implant surgery field, at least partly due to a significant number of procedures performed in the private environment; it is accordingly complicated to pursue negotiations with Health Authorities, for instance to improve reimbursement for this surgery.

Two key interventions appear to be strongly needed to change the present landscape of limited access by patients (pts) that qualify for penile prosthesis surgery. Firstly, to produce reliable data on the phenomenon "penile implant surgery" at National levels, and possibly on the benefits that this surgical intervention produces on recipients quality of life (QoL); secondly, to soundly inform the lay public of the penile prosthesis option existence, of the appropriate indications for this kind of surgery, and of real pros and cons. The Italian Society of Andrology, i.e. "Società Italiana di Andrologia" (S.I.A.), launched the "Registry project" on December 2014 (4) to address both those interventions: the project comprises in fact both a prospective Registry for penile implants, the Italian Nationwide Systematic Inventarisation of Surgical Treatment for ED (INSIST-ED) Registry, and a Institutional informative website aimed to the lay public: www.androprotesi.it. Given the short life of the INSIST-ED Registry, the purpose of this first report is to present a baseline data analysis of the characteristics of penile implant surgery in Italy.

\section{Material AND MEthods}

\section{Registry Project Board and Registry Coordinator}

S.I.A. Executive Committee appointed a Project Board (EP, GF, ECa, FC, FD) that has the tasks to evaluate before approval all the requests of implanting surgeons to adhere to the Registry, to deal with potential issues arising in the Registry, and to overview for ethics all the materials submitted by surgeons for the informative website www.androprotesi.it (see below).

The Project Coordinator (EP) directly interacts with the Project Monitor for the best functioning of the Registry.

\section{Registry characteristics}

The INSIST-ED Registry is a prospective registry of penile prostheses (all brands, all models) open to all implanting surgeons operating in Italy. Registry surgeons agree to provide anonymous patient, device, surgical procedure, outcomes, follow-up (FU) data, for both first and revision surgeries.

They also agree that device Companies could send the Registry Monitor (see below) periodic reports of number and type of implants performed by each specific surgeon since the date of his/her adhesion to the Registry. The Registry database has the following structure: surgery data, data at 1 month FU with report of possible complications, 1 year FU with data on patient QoL by means of the QoLSPP questionnaire (5), optional further FUs. Details of the database fields are reported in Supplementary Materials posted in www.aiua.it.

The INSIST-ED Registry can be accessed in its demo version at www.registro.andrologiaitaliana.it (username: demo@registrosia.it; password:RegSia2015_demo).

\section{Data analysis}

Registry data have been organized in a dedicated Excel format (Microsoft Excel 2011 for Mac, version 14.6.3); mean and standard deviation (SD) have been calculated.

\section{Registry Monitor}

A Registry Monitor (LU) has been appointed by the Registry Board. Monitor tasks are: to interact with new Registry implanting surgeons briefing and supporting them on data entry, to interact with device Companies to verify the completeness of number of surgeries inserted by implanting surgeons.

\section{Privacy protection and ethical issues}

The Registry protects patient privacy; no sensible data (i.e. patient name, date of birth, address, etc.) are present in the Registry.

For the protection of the privacy of each implanting surgeon individual surgical data inserted in the Registry can be accessed solely by the National Registry Coordinator and the Registry Monitor; Registry data are elaborated and circulated in a aggregated fashion only: no implanting surgeon can access data produced by individual implanting sur- geons. Similarly, it has been the choice of the Registry Board, on behalf of S.I.A., to report device data in an aggregated fashion only: three-component hydraulic devices, two-component hydraulic devices, non-hydraulic devices.

The "Registry Project" has been evaluated, before approval, by S.I.A, legal office, in particular under the profile of the patient privacy and anonymity protection law, and it has been found sound in this perspective.

\section{Institutional informative website}

S.I.A. concurrently created an Institutional website (www.androprotesi.it) aimed to provide lay public with sound information on the penile prosthesis option; only implanting surgeons adhering to the Registry and active on it (i.e. with at least one case entered in the Registry) are present in such website with a personal page.

The term "Institutional" refers to the fact that the Project Board, appointed by the scientific Society S.I.A., overviews the contents present in the informative website.

The purpose of www.androprotesi.it is both to correctly inform the lay public regarding the penile prosthesis option, and to motivate implanting surgeons to adhere to the Registry, as only surgeons active in the Registry are eligible to be present in the website with a personal page and contributions.

\section{Results}

The INSIST-ED Registry as by April 8, 2016 accounts for 31 implanting surgeons active in the Registry, and for 367 surgical procedures in the Registry database.

\section{Geographic distribution of performed surgeries}

The overall 367 surgical procedures related to penile prostheses resulted scattered through Italy in the following fashion: North $=139$ cases (Piedmont n. 70, Lombardy n. 8, Friuli n. 5, Veneto n. 8, Emilia-Romagna n. 48); Centre = 139 cases (Tuscany n. 30, Marche n. 26, Lazio n. 83); 


\section{Figure 1.}

Geographic distribution of performed surgeries.

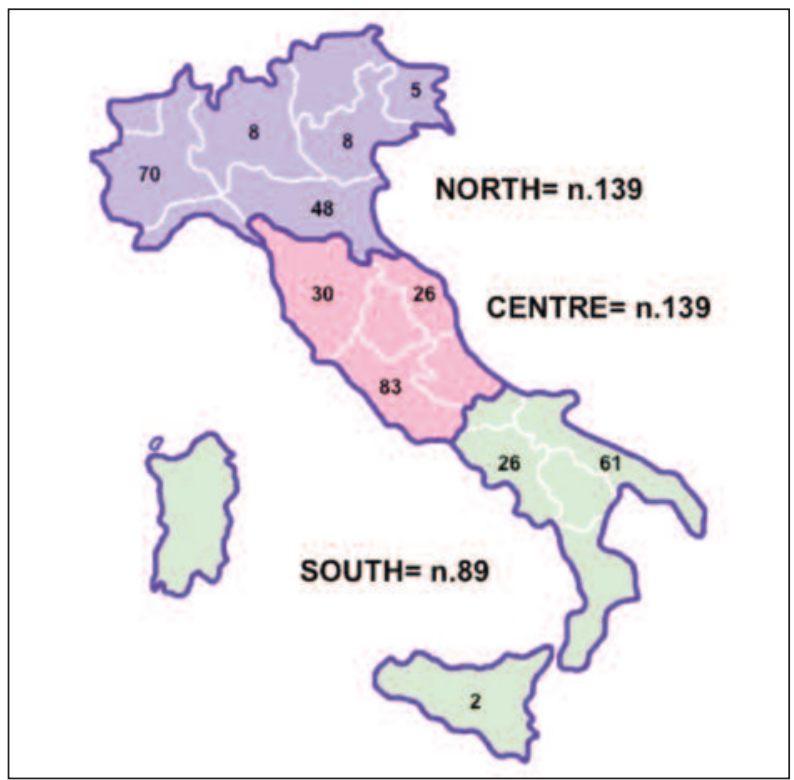

South $=89$ cases (Campania n. 26, Puglia n. 61, Sicily n. 2) (Figure 1).

Patient characteristics (age, type of surgery, and ED etiology) Age and type of surgery. The mean age of the overall 367 pts is 60,5 years (SD 9,6). Of them 310 pts underwent first penile implant surgery; they have a mean age of 60,4 years (SD 9,2); 43 patients underwent device explant and substitution (mean age: 59,6; SD 11). Fourteen patients underwent device explant only (mean age 63,6; SD 13) (Figure 2).

ED etiology. The 310 patients undergoing first penile implant surgery have the following primary diagnoses for their ED: status-post (s/p) radical pelvic surgery $n$. 111 (35,8\%), Peyronie's disease n. 66 (21,3\%), diabetes n. $39(12,6 \%)$, vascular disease n. 29 (9,4\%), neuropathy n. $5(1,6 \%)$, other n. $60(19,3 \%)$.

Surgery data (device type, surgical approach, reasons for revision, intraoperative complications)

Device type. The overall 367 surgeries comprise: implant of three-component hydraulic devices in 288 cases

\section{Table 1.}

Models of implanted devices.

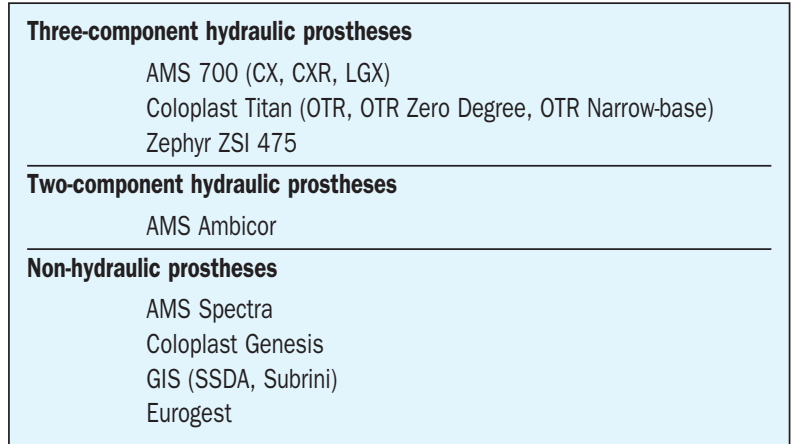

(78,5\%), implant of two-component hydraulic prostheses in 20 cases $(5,4 \%)$, implant of non-hydraulic prostheses in 45 cases $(12,3 \%)$, device explant only in 14 cases $(3,8 \%)$. The models of implanted devices are reported in Table 1.

In patients undergoing a first implant (n. 310), devices comprise: three-component hydraulic prostheses in 252 cases $(81,3 \%)$, two-component hydraulic prostheses in 16 cases $(5,2 \%)$, non-hydraulic prostheses in 42 cases $(13,5 \%)$. In the 43 device substitution cases the newly implanted devices comprise: three-component hydraulic prostheses in 36 cases $(83,7 \%)$, two-component hydraulic prostheses in 3 cases (7\%), non-hydraulic prostheses in 4 cases $(9,3 \%)$.

Surgical approach. In patients undergoing a first implant with a three-component hydraulic prosthesis (n. 252) the surgical approach is penoscrotal in 132 cases $(52,4 \%)$, infrapubic in 53 cases (21\%). Type of approach information are missing in 67 cases $(26,6 \%)$. A single incision is performed in 196 cases $(77,8 \%)$, a second incision in 40 cases $(15,9 \%)$, incision information are missing in 16 cases $(6,3 \%)$

Reasons for revision. Device substitution occurred in 43 patients, for the following reasons: fluid loss (n. 19), patient dissatisfaction (n. 6), erosion (n. 5), mechanical failure (n. 5), infection (n. 3), cylinder aneurism (n. 2), crossover (n. 1), reason not reported (n. 2).

Device explant without substitution occurred in 14 patients, for the following reasons: erosion (n. 6), infection (n. 4), mechanical failure (n. 4).

Intraoperative complications. In first penile implant surgeries (n. 310) intraoperative complications occurred in 2 cases $(0,6 \%)$ : one cross-over (intra-operatively recognized and corrected), and urethral lesion (surgery concluded with placement of a single cylinder only).

\section{Surgery focus 1: first penile implant in patients $s / p$ radical pelvic surgery (n. 111)}

These pts received a hydraulic device in 94 cases $(84,7 \%)$, two-component hydraulic prostheses in 9 cases $(8,1 \%)$, non-hydraulic prostheses in 8 cases $(7,2 \%)$.

Patients receiving a three-component prosthesis (n. 94). Surgical approach resulted penoscrotal in 80 cases $(85,1 \%)$ and infrapubic in 14 cases $(14,9 \%)$; reservoir was placed in Retzius space in 67 cases $(71,3 \%)$, intraperitoneally in 15 cases (15,9\%), ectopically in 12 cases $(12,8 \%)$. A single incision was performed in 61 cases $(64,9 \%)$, a second incision in the remaining 33 cases $(35,1 \%)$. Penile curvature was present at surgery in 13 patients $(13,9 \%)$, was not present in 77 patients $(81,9 \%)$, information missing in 4 cases $(4,2 \%)$.

\section{Surgery focus 2: first penile implant in patients with Peyronie's disease (n. 66)}

These patients received a hydraulic device in 50 cases (75,8\%), non-hydraulic prostheses in 16 cases $(24,2 \%)$; in no case a two-component hydraulic prosthesis was implanted.

Patients receiving a three-component prosthesis (n. 50). A single incision was performed in 36 cases (72\%), a second incision in the remaining 14 cases (28\%). Concerning intraoperative strategies aimed at curvature 


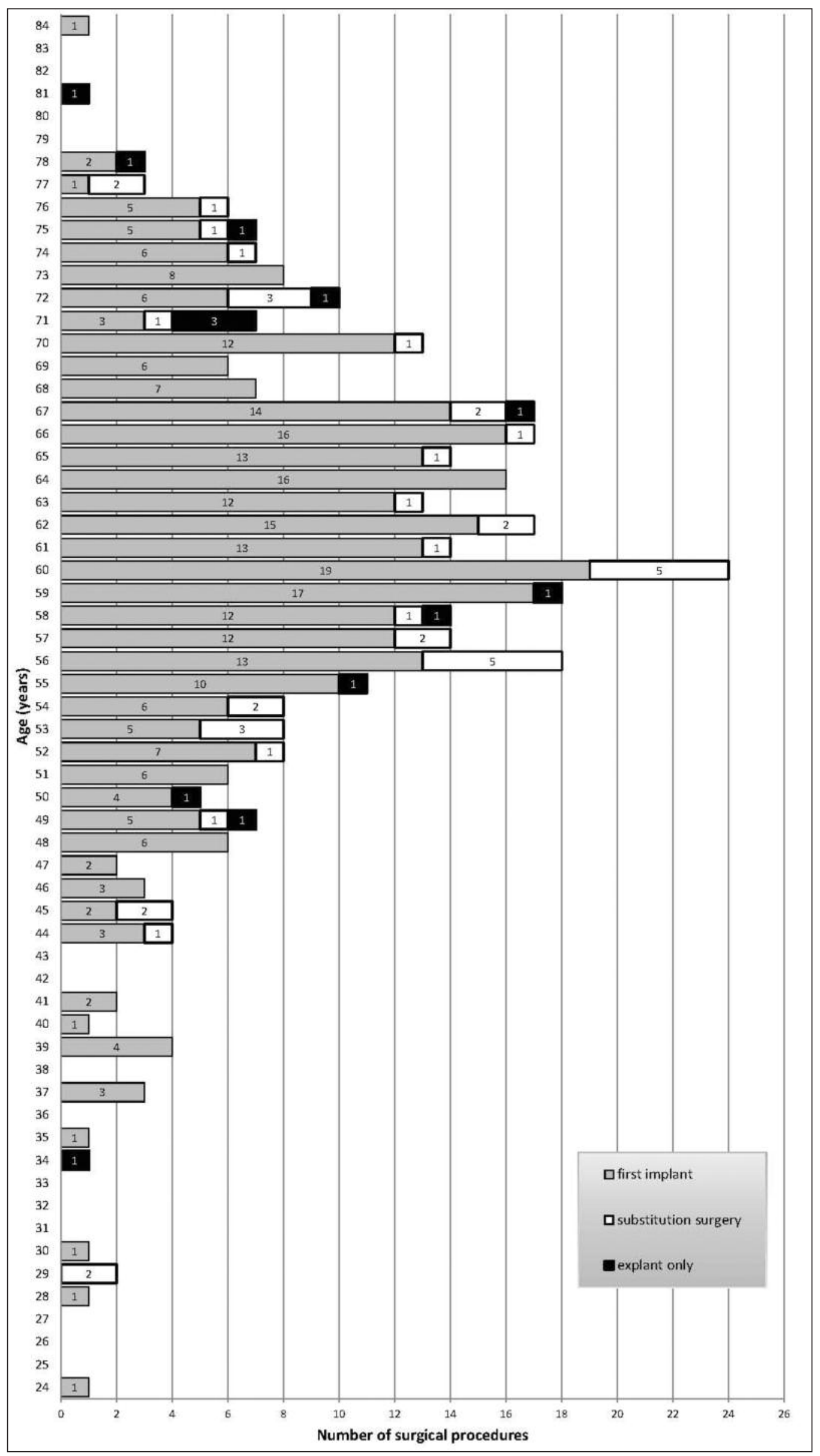

Figure 2.

Ages of

the 367 patients

in the three

groups first implant,

substitution

surgery,

and explant only. 
correction, Wilson maneuver (6) was performed in 31 cases $(72 \%)$, relaxing corporotomies without defect coverage were performed in 7 cases (14\%), relaxing corporotomies with defect coverage were performed in 6 cases $(12 \%)$; in 6 cases $(12 \%)$ no intraoperative procedure was needed.

\section{Surgery settings (public vs. private) and respective waiting times from indication to surgery}

Patients that underwent first penile implant (n. 310) had their surgery performed in public hospitals in 251 cases $(81 \%)$, in private settings in the remaining 59 cases (19\%). Waiting time from indication to surgery resulted: 8,2 months (SD 6,8) in public hospitals, 2,3 months (SD 6,2 ) in private settings. Patients treated in private settings paid out of their pocket in 49 cases (83\%), had insurance coverage in 10 cases (17\%).

\section{Discussion}

The INSIST-ED Registry is the first European experience of a Registry devoted to penile implants. It provides for the first time a real-world picture of penile implant surgery in Italy, thanks to the adhesion of surgeons performing penile implants (all brands, all types, all models) in Italy, with no restrictions in terms of surgeon implant volumes and of Society affiliation.

Launched on December 2014 by the Italian Society of Andrology (1), the Registry as by April 8, 2016 has been joined by 31 active implanting surgeons, and accounts for 367 procedures inserted in the database.

The short life of the INSIST-ED Registry at present allows for a baseline data analysis only, but the structure of the Registry should provide shortly data also on surgical outcomes, postoperative complications, follow-up. Presently, the only other existing Registry of penile implants is the U.S.A. PROPPER Registry (7).

The two registries have several structural differences, chief ones being: PROPPER addresses American Medical Systems (AMS) penile implants only, AMS is responsible for the database and statistical analysis, implanting surgeons have been selected among high volume implanting surgeons; the INSIST-ED Registry addresses all brands/types/models of penile implants, the scientific Society S.I.A. is responsible for the database and statistical analysis, all implanting surgeons operating in Italy could adhere to the Registry regardless of their implant volumes. Such differences mandate caution in making comparisons between the outcomes of the two registries, nonetheless some general considerations can be proposed.

The first data analysis of the INSIST-ED Registry shows that the geographic distribution of the performed procedures is overall even across the three Italian macro-areas of North, Centre, South. Distribution becomes anyhow uneven when considering the different regions: 7 regions have 26-70 procedures in the Registry, 4 regions have 2-8 procedures, 11 regions have none. This may reflect both a selective adhesion of implanting surgeons to the Registry, and/or a real different regional availability/access to the penile prosthesis option across different Italian regions.

Mean age of patients in our series is 60 years (SD 9,6), that appears similar to that recorded in the PROPPER study (mean 63,6; SD 10).

The leading primary causes for severe ED in first implant surgeries were: former radical pelvic surgery $(35,8 \%)$, Peyronie's disease $(21,3 \%)$ and diabetes $(12,6 \%)$, respectively. In this aspect there is an apparent difference with data of the PROPPER study that describe as leading ED causes, besides former radical prostatectomy (28\%): diabetes $(21 \%)$, cardiovascular disease $(19,6 \%)$, and Peyronie's disease $(8,9 \%)$. We suggest that such differences may reflect the different prevalence of metabolic syndrome in Italy and U.S.A. $(8,9)$.

The most popular device type resulted the three-component hydraulic prosthesis in all the patient groups considered (overall, first implant, substitution, subgroup of $\mathrm{s} / \mathrm{p}$ radical pelvic surgery, Peyronie's disease), followed by non-hydraulic devices, with the exception of the subgroup s/p radical pelvic surgery, where two-component hydraulic prostheses were by little preferred over nonhydraulic prostheses. Two-component hydraulic prostheses were never used in Peyronie's cases. Also in the PROPPER study the three-component model was the most popular, but to a higher extent $(96,5 \%)$ compared to the INSIST-ED registry $(83,7 \%$ in first implant cases). Differently from PROPPER we record in Italy a certain degree of popularity for the non-hydraulic models: $13,5 \%$ in first implant surgery versus $1 \%$ in U.S.A.. Such difference might reflect both: differences in implanting surgeons (in INSIST-ED: not selected according to surgery volume, i.e. possibly including also less experienced surgeons), and device costs: in Italy three-component hydraulic prostheses are marketed with a roughly double price compared to the U.S.A.

The preferred surgical approach for three-component devices (first implant) resulted by far the penoscrotal one, with a single incision only, similar to the PROPPER study.

In cases of former radical pelvic surgery the reservoir of three-pieces devices has been placed ectopically in the minority of cases, as in PROPPER, but to a lesser extent (12,8\% in INSIST-ED vs. $31,8 \%$ in PROPPER). In such cases, i.e. $\mathrm{s} / \mathrm{p}$ radical pelvic surgery, penile curvature was observed in $13,9 \%$ of cases, consistently with the report of Tal et al. (10).

In cases of Peyronie's disease the most popular approach to solve the curvature resulted the Wilson maneuver (6). In the 310 first penile implant surgeries intraoperative complications occurred in 2 cases $(0,6 \%)$ only, implying that implanting surgeons that adhere to the INSIST-ED Registry are appropriately skilled.

Majority of first implants were performed in public settings (81\%), but with a significant waiting time from indication to surgery. Furthermore, the vast majority of patients undergoing surgery in private settings were not covered by medical insurance. It accordingly appears that there is a definite need in Italy for better Public Health policies for the penile implant surgery area; for instance, more favorable reimbursements and more prosthesis units/year available in public hospitals. We hope that present and future outcomes of the INSIST-ED Registry will be instrumental in negotiations with Health Authorities on such aspects. 


\section{Conclusions}

The INSIST-ED Registry represents the first European experience of penile prosthesis Registry; it has the purpose to produce objective, real world hard data on the landscape of penile implant surgery in Italy.

The first baseline analysis of 367 procedures entered in the Registry by 31 implanting surgeons operating in Italy shows chiefly that three-pieces inflatable prosthesis is the most implanted device, the leading etiology of ED in patient receiving a prosthesis is former radical pelvic surgery, the primary reason for revision surgery is device failure, primary settings for first penile implant surgery are public hospitals.

We do hope that the first experiences of penile prosthesis surgery registries, INSIST-ED in Italy and PROPPER in U.S.A., will be soon followed by other Countries, so to generate a great amount of prospective, multicenter, multinational, comparable data for the benefit of patients and physicians: better Public Health policies and definition of the best surgical standards.

\section{ACKNOWLEDGMENT}

Authors wish to thank Dott. Ilaria Stanghellini and Dr. Alberto Strano for their support in statistical data analysis.

\section{REFERENCES}

1. Rajpurkar A,Dhabuwala CB. Comparison of satisfaction rates and erectile function in patients treated with sildenafil, intracav- ernous prostaglandin E1 and penile implant surgery for erectile dysfunction in urology practice. J Urol. 2003; 170:159-63.

2. Bernal RM, Henry GD. Contemporary Patient Satisfaction Rates for Three-Piece Inflatable Penile Prostheses. Advances in Urology, 2012, article ID 707321, http://dx.doi.org/10.1155/2012/707321.

3. Parazzini F, Menchini Fabris F, Bortolotti A, et al. Frequency and determinants of erectile dysfunction in Italy. Eur Urol. 2000; 37:43-9.

4. Pescatori E, Franco G. Introducing the first Registry for penile implants: the INSIST-ED Registry (Italian Nationwide Systematic Inventarisation of Surgical Treatment for ED). J Sex Med 2015; 12(suppl 3):216.240 HP-06-005

5..Caraceni E, Utizi L. A questionnaire for the evaluation of quality of life after penile prosthesis implant: quality of life and sexuality with penile prosthesis (QoLSPP): to what extent does the implant affect the patient's life? JSM 2014; 11:1005-12.

6. Wilson SK, Delk JR. A new treatment for Peyronie's disease: modeling the penis over an inflatable penile prosthesis. J Urol. 1994; 152:1121-1123.

7. Henry GD, Karpman E, Brant W, et al. The Who, How and What of Real-World Penile Implantation in 2015: The PROPPER Registry Baseline Data. J Urol. 2016; 195:427-33.

8. Balkau B, Charles MA, Drivsholm T, et al. Frequency of the WHO metabolic syndrome in European cohorts, and an alternative definition of an insulin resistance syndrome. Diabet Metab 2002; 28:364-76.

9. Ford ES, Giles WH, Dietz WH. Prevalence of the metabolic syndrome among US adults: findings from the Third National Health and Nutrition Examination Survey. JAMA 2002; 287:356-9.

10. Tal R, Heck M, Teloken P, et al. Peyronie's Disease Following Radical Prostatectomy: Incidence and Predictors. J Sex Med. 2010; 7:1254-1261.

\section{Correspondence}

Edoardo Pescatori, MD (Corresponding Author)

info@andrologiapescatori.it - Hesperia Hospital, Modena

Giovanni Alei, MD

info@giovannialei.it - Policlinico Umberto I, Roma

Gabriele Antonini, MD

dottgabrieleantonini@gmail.com - Policlinico Umberto I, Roma

Antonio Avolio, MD

antonio.avolio@gmail.com - Ospedale Mazzoni, Ascoli Piceno

Carlo Bettocchi, MD

carlo.bettocchi@uniba.it - Policlinico Bari

Marco Bitelli, MD

marcobitelli@yahoo.com - Ospedale S. Sebastiano Martire, Frascati

Francesco Boezio, MD

francescoboezio@alice.it - Ospedale Tatarella, Cerignola

Tommaso Cai, MD

ktommy@libero.it - Ospedale Santa Chiara, Trento

Enrico Caraceni, MD

ecarace1@gmail.com - Area Vasta 3, Civitanova Marche

Maurizio Carrino, MD

cris63@libero.it - Ospedale Cardarelli, Napoli

Fulvio Colombo, MD

fulvio.colombo@aosp.bo.it - Policlinico S.Orsola-Malpighi, Bologna

Enrico Conti, MD

econti@aslcn2.it - Ospedale San Bartolomeo, Sarzana

Antonio Corvasce, MD

a.corvasce@katamail.com - Ospedale Bonomo, Andria

Federico Dehò, MD

deho.federico@gmail.com - San Raffaele, Milano

Stefano Fiordelise, MD

stfiorde@tin.it - AUSL Piacenza

Nicola Ghidini, MD

Hesperia Hospital, Modena - info@nicolaghidini.it

Emilio Italiano, MD

eitaliano@gmail.com - Ospedali Riuniti, Palermo

Giuseppe La Pera, MD

lapera@libero.it - San Camillo Forlanini, Roma
Giovanni Liguori, MD

gioliguori33@gmail.com - Ospedali Riuniti, Trieste

Carlo Maretti, MD

carlomaretti@tin.it - CIRM, Piacenza

Nicola Mondaini, MD

info@nicolamondaini.it - Ospedale S.M. Annunziata, Firenze

Alessandro Natali, MD

info@profnatali.it - AUOC, Firenze

Carlo Negro, MD

carlo.negro@gmail.com - Ospedale Cardinal Massaia, Asti

Alessandro Palmieri, MD

info@alessandropalmieri.it - Federico II, Napoli

Fabrizio Palumbo, MD

palumbo.fab@gmail.com - Policlinico Bari

Matteo Paradiso, MD

m.paradiso2014@gmail.com - Ospedale Cardinal Massaia, Asti

Massimo Polito, MD

max_polito@virgilio.it - Ospedali Riuniti, Ancona

Diego Pozza, MD

diegopo@tin.it - Wojtyla Hospital, Roma

Mauro Silvani, $M D$

dr.silvani@libero.it - Ospedale Infermi, Biella

Aldo Tamai, MD

aldotamai@libero.it - Casa di Cura Eretenia, Vicenza

Massimiliano Timpano, MD

info@andrologiatorino.it - Ospedale Molinette, Torino

Lilia Utizi, MD

lilia16@libero.it - Area Vasta 3, Civitanova Marche

Francesco Varvello, MD

francesco.varvello@me.com - Ospedale San Lazzaro, Alba

Patrizio Vicini, MD

patriziovicini@inwind.it - Rome American Hospital, Roma

Antonio Vitarelli, MD

antoniovitarelli@hotmail.com - Policlinico Bari

Giorgio Franco, MD

giorgio.franco@libero.it - Policlinico Umberto I, Roma 